\title{
Automorphism groups of maps, hypermaps and dessins
}

\author{
Gareth Aneurin Jones* \\ School of Mathematical Sciences, University of Southampton, \\ Southampton SO17 1BJ, UK
}

Received 5 November 2018, accepted 2 September 2019, published online 5 August 2020

\begin{abstract}
A detailed proof is given of a theorem describing the centraliser of a transitive permutation group, with applications to automorphism groups of objects in various categories of maps, hypermaps, dessins, polytopes and covering spaces, where the automorphism group of any object is the centraliser of its monodromy group. An alternative form of the theorem, valid for finite objects, is discussed, with counterexamples based on Baumslag-Solitar groups to show how it can fail in the infinite case. The automorphism groups of objects with primitive monodromy groups are described, as are those of non-connected objects.
\end{abstract}

Keywords: Permutation group, centraliser, automorphism group, map, hypermap, dessin d'enfant.

Math. Subj. Class. (2020): 05C10, 14H57, 20B25, 20B27, 52B15, 57M10

\section{Introduction}

In certain categories $\mathfrak{C}$, such as those consisting of maps or hypermaps, oriented or unoriented, or of dessins d'enfants (regarded as finite oriented hypermaps), each object $\mathcal{O}$ can be identified with a permutation representation $\theta: \Gamma \rightarrow S:=\operatorname{Sym}(\Omega)$ of a 'parent group' $\Gamma=\Gamma_{\mathfrak{C}}$ on some set $\Omega$, and the morphisms $\mathcal{O}_{1} \rightarrow \mathcal{O}_{2}$ can be identified with the functions $\Omega_{1} \rightarrow \Omega_{2}$ which commute with the actions of $\Gamma$ on the corresponding sets $\Omega_{1}$ and $\Omega_{2}$. These are the 'permutational categories' defined and discussed in [9] (see also $\S 2)$. The automorphism group $\operatorname{Aut}_{\mathfrak{C}}(\mathcal{O})$ of an object $\mathcal{O}$ within $\mathfrak{C}$ is then identified with the centraliser $C:=C_{S}(G)$ in $S$ of the monodromy group $G:=\theta(\Gamma)$ of $\mathcal{O}$. Now $\mathcal{O}$ is connected if and only if $G$ is transitive on $\Omega$, as we will assume unless otherwise stated (see §6). In this situation, an important result is the following, where $N$ denotes the normaliser of a subgroup:

\footnotetext{
*The author is grateful to Ernesto Girondo, Gabino González-Diez and Rubén Hidalgo for discussions about dessins d'enfants which motivated this work.

E-mail address: g.a.jones@ maths.soton.ac.uk (Gareth Aneurin Jones)
} 
Theorem 1.1. If $\mathcal{O}$ is connected then

$$
\operatorname{Aut}_{\mathfrak{C}}(\mathcal{O}) \cong N_{G}(H) / H \cong N_{\Gamma}(M) / M
$$

where $H$ and $M$ are the stabilisers in $G$ and $\Gamma$ of some $\alpha \in \Omega$, and $N_{G}(H)$ and $N_{\Gamma}(M)$ are their normalisers.

For instance, this theorem has recently been used in [10] to show that in various categories of maps and hypermaps, every countable group can be realised as the automorphism group of uncountably many non-isomorphic objects, infinitely many of which can be chosen to be finite if the group is. This is an analogue of the well-known theorems of Frucht [5] and Sabidussi [20] for graphs and their automorphism groups.

There are analogues of Theorem 1.1 in other contexts, ranging from abstract polytopes to covering spaces. Proofs of Theorem 1.1 for particular categories can be found in the literature: for instance, in [11] it is deduced for oriented maps from a more general result about morphisms in that category; in [12, Theorem 2.2 and Corollary 2.1] a proof for dessins is briefly outlined; analogous results for covering spaces are proved in [15, Appendix] and [17, Theorem 81.2], and for abstract polytopes in [16, Propositions 2D8 and 2E23(a)]. In $\S 3$ we give a detailed proof of the following 'folklore' theorem about permutation groups (see also Theorem 3.2 in the recent [19]), which immediately implies Theorem 1.1 for all permutational categories, including the special cases listed above.

Theorem 1.2. Let $G$ be a transitive permutation group on a set $\Omega$, with $H$ the stabiliser of some $\alpha \in \Omega$, and let $C:=C_{S}(G)$ be the centraliser of $G$ in the symmetric group $S:=\operatorname{Sym}(\Omega)$. Then

\section{1. $C \cong N_{G}(H) / H$,}

2. $C$ acts regularly on the set $\Phi$ of elements of $\Omega$ with stabiliser $H$.

One sometimes finds proofs or statements of particular cases of Theorem 1.2 which include the assertion that $C$ acts regularly on the set $\Phi$ of fixed points of $H$ in $\Omega$; while this is valid if $H$ is finite, in $\S 4$ we give counter-examples, based on Baumslag-Solitar groups [1], to show that if $H$ is infinite then $\Phi$ must be redefined more precisely as in (2). It follows from Theorem 1.1 that if the monodromy group $G$ of an object $\mathcal{O}$ acts primitively on $\Omega$, then either $\operatorname{Aut}_{\mathfrak{C}}(\mathcal{O})$ is trivial, or $G$ is a cyclic group of prime order, acting regularly on $\Omega$; in $\S 5$ we describe the objects with the latter property in various categories $\mathfrak{C}$. In $\S 6$ we briefly consider the structure and cardinality of the automorphism groups of non-connected objects in permutational categories, and in $\S 7$ we extend Theorem 1.1 to cover morphisms between connected objects.

\section{Permutational categories}

A permutational category $\mathfrak{C}$ is defined in [9] to be a category in which the objects $\mathcal{O}$ can be identified with the permutation representations $\theta: \Gamma \rightarrow S:=\operatorname{Sym}(\Omega)$ of a parent group $\Gamma=\Gamma_{\mathfrak{C}}$, and the morphisms $\mathcal{O}_{1} \rightarrow \mathcal{O}_{2}$ with the $\Gamma$-invariant functions $\Omega_{1} \rightarrow \Omega_{2}$, those commuting with the actions of $\Gamma$ on $\Omega_{1}$ and $\Omega_{2}$. The automorphism group $\operatorname{Aut}(\mathcal{O})=$ $\operatorname{Aut}_{\mathfrak{C}}(\mathcal{O})$ of $\mathcal{O}$ in the category $\mathfrak{C}$ is then the group of all permutations of $\Omega$ commuting with $\Gamma$, that is, the centraliser $C_{S}(G)$ of the monodromy group $G=\theta(\Gamma)$ of $\mathcal{O}$ in the symmetric group $S$. Here we will restrict our attention to the connected objects, those for which $\Gamma$ acts transitively on $\Omega$. 
We will concentrate mainly on five particular examples of permutational categories, outlined briefly below (for full details, and other examples, see [9]). In each case, the parent group $\Gamma$ is either an extended triangle group

$$
\Delta[p, q, r]=\left\langle R_{0}, R_{1}, R_{2} \mid R_{i}^{2}=\left(R_{1} R_{2}\right)^{p}=\left(R_{2} R_{0}\right)^{q}=\left(R_{0} R_{1}\right)^{r}=1\right\rangle,
$$

or its orientation-preserving subgroup of index 2 , the triangle group

$$
\Delta(p, q, r)=\left\langle X, Y, Z \mid X^{p}=Y^{q}=Z^{r}=X Y Z=1\right\rangle,
$$

where $X=R_{1} R_{2}, Y=R_{2} R_{0}$ and $Z=R_{0} R_{1}$. Here $p, q, r \in \mathbb{N} \cup\{\infty\}$, and we ignore any relations of the form $W^{\infty}=1$. In what follows, $*$ denotes a free product, $C_{n}$ denotes a cyclic group of order $n \in \mathbb{N} \cup\{\infty\}$, while $V_{n}$ is an elementary abelian group of order $n$, and $F_{r}$ is a free group of rank $r$.

1. The category $\mathfrak{M}$ of maps on surfaces (possibly non-orientable or with boundary), that is, embeddings of graphs with simply connected faces, has parent group

$$
\Gamma=\Gamma_{\mathfrak{M}}=\Delta[\infty, 2, \infty] \cong V_{4} * C_{2} .
$$

This permutes the set $\Omega$ of incident vertex-edge-face flags of a map (equivalently, the faces of its barycentric subdivision), with each involution $R_{i}(i=0,1,2)$ changing the $i$-dimensional component of each flag (whenever possible) while preserving the other two.

2. The category $\mathfrak{M}^{+}$of oriented maps, those in which the underlying surface is oriented and without boundary, has parent group

$$
\Gamma=\Gamma_{\mathfrak{M}^{+}}=\Delta(\infty, 2, \infty) \cong C_{\infty} * C_{2}
$$

This group permutes directed edges, with $X$ usng the local orientation to rotate them about their target vertices, and $Y$ reversing their direction, so that $Z$ rotates them around incident faces.

3. There are several ways of defining or representing hypermaps. For our purposes, the most convenient is the Walsh representation as a bipartite map [21], in which the black and white vertices of the embedded graph correspond to the hypervertices and hyperedges of the hypermap, the edges correspond to incidences between them, and the faces correspond to its hyperfaces. The category $\mathfrak{H}$ of all hypermaps, where the underlying surface is unoriented and possibly with boundary, has parent group

$$
\Gamma=\Gamma_{\mathfrak{H}}=\Delta[\infty, \infty, \infty] \cong C_{2} * C_{2} * C_{2} .
$$

This permutes incident edge-face pairs of the bipartite map, with involutions $R_{0}$ and $R_{1}$ preserving the face and the incident white and black vertex respectively, while $R_{2}$ preserves the edge.

4. The category $\mathfrak{H}^{+}$of oriented hypermaps, those in which the underlying surface is oriented and without boundary, has parent group

$$
\Gamma=\Gamma_{\mathfrak{H}^{+}}=\Delta(\infty, \infty, \infty) \cong C_{\infty} * C_{\infty} \cong F_{2} .
$$

This permutes the edges of the embedded graph, with $X$ and $Y$ using the local orientation to rotate them around their incident black and white vertices, so that $Z$ rotates them around incident faces. 
5. The category $\mathfrak{D}$ of dessins d'enfants can be identified with the subcategory of $\mathfrak{H}^{+}$consisting of its finite objects, those in which the embedded bipartite graph is finite and the surface is compact. It has the same parent group $\Gamma=\Delta(\infty, \infty, \infty) \cong F_{2}$ as $\mathcal{H}^{+}$, permuting edges as before.

If we wish to restrict any of these categories to the subcategory of objects of a particular type $(p, q, r)$, we replace the parent group given above with the corresponding triangle or extended triangle group of that type.
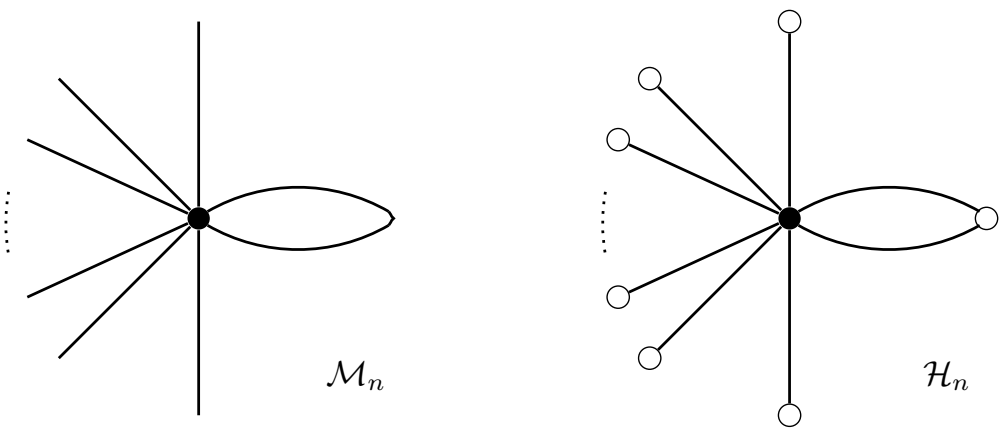

Figure 1: The map $\mathcal{M}_{n}$ and the hypermap $\mathcal{H}_{n}$

Example 2.1. The planar map $\mathcal{M}_{n} \in \mathfrak{M}^{+}(n \geq 2)$, shown on the left in Figure 1, has one vertex, of valency $n$, incident with one loop and $n-2$ half edges. It corresponds to the epimorphism $\theta: \Delta(\infty, 2, \infty) \rightarrow S_{n}$ given by

$$
X \mapsto x=(1,2, \ldots, n), \quad Y \mapsto y=(1,2), \quad Z \mapsto z=(n, n-1, \ldots, 2) .
$$

This map can be regarded as a hypermap $\mathcal{H}_{n}$, shown on the right in Figure 1, by adding a white vertex to each edge; this corresponds to composing $\theta$ with the natural epimorphism $\Delta(\infty, \infty, \infty) \rightarrow \Delta(\infty, 2, \infty)$. The hypermap $\mathcal{H}_{n}$ has type $(n, 2, n-1)$, and it can be regarded as a member of the category of oriented hypermaps of this type by factoring $\theta$ through $\Delta(n, 2, n-1)$. In all cases the monodromy group $G$ is $S_{n}$, in its natural representation, and the automorphism group is trivial. However, if we regard $\mathcal{M}_{n}$ or $\mathcal{H}_{n}$ as an unoriented map or hypermap, then its monodromy group is $S_{n} \times S_{2}$ in its natural product action of degree $2 n$, and the automorphism group has order 2 , generated by the obvious reflection.

We briefly mention two other classes of permutational categories in which Theorem 1.1 applies. The first concerns abstract polytopes [16], regarded as higher-dimensional generalisations of maps. Those $n$-polytopes of a particular type, associated with the Schläfli symbol $\left\{p_{1}, \ldots, p_{n-1}\right\}$, can be identified with transitive permutation representations of the Coxeter group $\Gamma$ with presentation

$$
\left\langle R_{0}, \ldots, R_{n} \mid R_{i}^{2}=\left(R_{i-1} R_{i}\right)^{p_{i}}=\left(R_{i} R_{j}\right)^{2}=1(|i-j|>1)\right\rangle .
$$

For instance, $\Gamma_{\mathfrak{M}}$ is associated with the Schläfli symbol $\{\infty, \infty\}$. However, in higher dimensions, not all transitive representations correspond to abstract polytopes, since the monodromy groups must satisfy the intersection property [16, Proposition 2B10]. 
The second class of examples concerns covering spaces [15, 17]. Under suitable connectedness assumptions, the (connected, unbranched) coverings $Y \rightarrow X$ of a topological space $X$ can be identified with the transitive permutation representations $\theta: \Gamma \rightarrow S=$ $\operatorname{Sym}(\Omega)$ of its fundamental group $\Gamma=\pi_{1} X$, acting by unique path-lifting on the fibre $\Omega$ over a base-point in $X$. In this case the automorphism group of an object $Y \rightarrow X$ in this category is the group of covering transformations, the centraliser in $S$ of the monodromy group $\theta(\Gamma)$ of the covering.

This last example helps to explain the importance of the fifth category listed above, the category $\mathfrak{D}$ of dessins d'enfants. By Belyi's Theorem [2], a compact Riemann surface $R$ is defined (as a projective algebraic curve) over the field $\overline{\mathbb{Q}}$ of algebraic numbers if and only if it admits a non-constant meromorphic function $\beta$ branched over at most three points of the complex projective line (the Riemann sphere) $\mathbb{P}^{1}(\mathbb{C})=\hat{\mathbb{C}}=\mathbb{C} \cup\{\infty\}$. Composing $\beta$ with a Möbius transformation if necessary, we may assume that its critical values are contained in $\{0,1, \infty\}$. Such Belyi functions $\beta$ correspond to unbranched finite coverings $R \backslash \beta^{-1}(\{0,1, \infty\}) \rightarrow X$ of the thrice-punctured sphere $X=\hat{\mathbb{C}} \backslash\{0,1, \infty\}$, and hence to transitive finite permutation representations of its fundamental group $\Gamma=\pi_{1} X$; this is a free group of rank 2 , freely generated by the homotopy classes of small loops around 0 and 1. The unit interval $[0,1] \subset \hat{\mathbb{C}}$ lifts to a bipartite graph embedded in $R$, with black and white vertices over 0 and 1 , and face-centres over $\infty$. Conversely, any finite oriented hypermap, after suitable uniformisation, yields a Riemann surface $R$ defined over $\overline{\mathbb{Q}}$; see $[6,7,12,13]$ for details of these connections, and of the action of the absolute Galois group $\operatorname{Gal}(\overline{\mathbb{Q}} / \mathbb{Q})$ on dessins.

\section{Proof of Theorems 1.1 and 1.2}

Let $\mathcal{O}$ be a connected object in a permutational category $\mathfrak{C}$, identified with a transitive permutation representation $\Gamma \rightarrow G \leq S:=\operatorname{Sym}(\Omega)$, so that its automorphism group $\operatorname{Aut}_{\mathfrak{C}}(\mathcal{O})$ is identified with the centraliser $C_{S}(G)$ of $G$ in $S$. Then Theorem 1.1 asserts that

$$
\operatorname{Aut}_{\mathfrak{C}^{\mathrm{C}}}(\mathcal{O}) \cong N_{G}(H) / H \cong N_{\Gamma}(M) / M
$$

where $G$ is the monodromy group of $\mathcal{O}$, and $H$ and $M$ are point-stabilisers in $G$ and $\Gamma$. The second isomorphism follows immediately from the first, and this in turn follows from part (1) of Theorem 1.2, both parts of which we will now prove.

Proof. The centraliser $C$ of $G$ acts semi-regularly (i.e. freely) on $\Omega$. To see this, suppose that $c \in C$ and $\beta c=\beta$ for some $\beta \in \Omega$. Given any $\omega \in \Omega$, there is some $g \in G$ such that $\omega=\beta g$, since $G$ is transitive on $\Omega$. Then $\omega c=(\beta g) c=(\beta c) g=\beta g=\omega$. Thus $c=1$, as required.

Let $\Phi=\left\{\beta \in \Omega \mid G_{\beta}=H\right\}$, so in particular $\alpha \in \Phi$. Then $C$ leaves $\Phi$ invariant, since if $\beta \in \Phi$ and $c \in C$ then for all $h \in H$ we have $(\beta c) h=(\beta h) c=\beta c$, so that $\beta c \in \Phi$.

Let us identify $\Omega$ with the set of cosets of $H$ in $G$ in the usual way, identifying each $\omega \in \Omega$ with the unique coset $H x$ such that $x \in G$ and $\alpha x=\omega$. Thus $\alpha$ is identified with $H$ itself, and $G$ acts on the cosets by $g: H x \mapsto H x g$.

Then $\Phi$ is identified with the set of cosets of $H$ in $N:=N_{G}(H)$. To see this, let $\omega \in \Omega$ correspond to a coset $H x$ of $H$ in $G$. First suppose that $x=n \in N$. Then $(H n) h=(n H) h=n(H h)=n H=H n$ for all $h \in H$, so the coset $H n$ is fixed by $H$, giving $H \leq G_{\omega}$, while if $g \in G_{\omega}$ then $H n g=H n$, so $H=H^{n}=H^{n} g=H g$ and 
hence $g \in H$, giving $G_{\omega} \leq H$. Thus $G_{\omega}=H$ and hence $\omega \in \Phi$. Conversely, suppose that $\omega \in \Phi$. Then $G_{\omega}=H$, so $H x g=H x$ if and only if $g \in H$, that is, $H^{x} g=H^{x}$ if and only if $g \in H$, so $H^{x}=H$ and hence $x \in N$.

Let us define a new action of $N$ on $\Omega$ (now regarded as the set of cosets of $H$ in $G$ ) by $n: H x \mapsto n^{-1} H x=H n^{-1} x$ for all $n \in N$ and $x \in G$. If $n_{1}, n_{2} \in N$ then $n_{1}$, followed by $n_{2}$, sends $H x$ to $n_{2}^{-1} n_{1}^{-1} H x=\left(n_{1} n_{2}\right)^{-1} H x$, as does $n_{1} n_{2}$, so this is indeed a group action of $N$. It commutes with the action of $G$ on $\Omega$, since $n^{-1}(H x g)=\left(n^{-1} H x\right) g$ for all $n \in N$ and $x, g \in G$, so it defines a homomorphism $\theta: N \rightarrow C$. In particular, this action preserves $\Phi$, since $C$ does.

The induced action of $N$ on $\Phi$ is transitive, since if $n^{\prime}, n^{\prime \prime} \in N$ then the element $n=n^{\prime}\left(n^{\prime \prime}\right)^{-1} \in N$ sends $H n^{\prime}$ to $n^{-1} H n^{\prime}=H n^{-1} n^{\prime}=H n^{\prime \prime}$. Thus $\theta(N)$ acts on $\Phi$ as a transitive subgroup of $C$. But $C$ acts semi-regularly on $\Phi$, so it has no transitive proper subgroups. Therefore $\theta$ is an epimorphism, and $C$ acts regularly on $\Phi$, giving (2).

In this action of $N$, we have $n^{-1} H x=H x$ if and only if $n \in H$, so the subgroup stabilising any coset $H x$ is $H$, which is therefore the kernel $\operatorname{ker}(\theta)$ of this action of $N$. The First Isomorphism Theorem therefore gives $N / H \cong C$, so (1) is proved.

Remark 3.1. The most symmetric objects in $\mathfrak{C}$ are the regular objects, those for which $\operatorname{Aut}_{\mathfrak{C}}(\mathcal{O})$ acts transitively on $\Omega$. By Theorem 1.2(2) this is equivalent to $\Phi=\Omega$, that is, to $H=1$, meaning that $G$ acts regularly on $\Omega$. This is also equivalent to $M$ being a normal subgroup of $\Gamma$. Then $G \cong C \cong \Gamma / M$, and $G$ and $C$ can be identified with the right and left regular representations of the same group. (In fact, if $G$ is abelian then $C=G$.)

Remark 3.2. A variety of groups is a class of groups defined by identical relations between their elements (see [18]); simple examples include groups of exponent dividing $n$, solvable groups of derived length at most $l$, and nilpotent groups of class at most $c$. The fact that the centraliser $C$ of a transitive permutation group $G$ can be realised within $G$ as $N_{G}(H) / H$ means that if $G$ is a member of a variety $\mathfrak{V}$, then so is $C$. This may seem surprising since, as subgroups of the symmetric group $S$, the groups $C$ and $G$ could have a very small intersection: for instance, $C \cap G=1$ if, as in many cases, $G$ has a trivial centre. Of course, this fact applies to permutational categories: if the parent group $\Gamma$ is in $\mathfrak{V}$ then the automorphism group of each connected object is also in $\mathfrak{V}$. However, for most of the examples we are interested in, $\Gamma$ generates the variety of all groups, and the only restrictions on automorphism groups are the obvious ones imposed by cardinality, as shown in [10].

\section{An alternative form of Theorem 1.2(2)}

One sometimes finds part (2) of Theorem 1.2 stated in the following alternative form:

$\left(2^{\prime}\right) C$ acts regularly on the set of fixed points of $H$ in $\Omega$.

This is equivalent to (2) in cases where $\Omega$ is finite, or more generally where $H$ is finite, so that an inclusion $H=H_{\alpha} \leq H_{\beta}$ between conjugate subgroups is equivalent to their equality. However, $\left(2^{\prime}\right)$ can be false if $H$ is infinite, as shown by the following example.

Example 4.1. Although our aim here is mainly group-theoretic, to construct a permutation group $G$ on a set $\Omega$ such that condition $\left(2^{\prime}\right)$ fails, the original motivation, as in much of this paper, is combinatorial. It may therefore be useful, throughout this construction, to think of $G$ as the monodromy group of an oriented hypermap $\mathcal{H}$, regarded as a bipartite map, with elements of $\Omega$ as edges, and cycles of the generators $a$ and $b$ of $G$ as black and white vertices (see Remark 4.2). 
Let $G$ be the Baumslag-Solitar group [1]

$$
G=B S(1,2)=\left\langle a, b \mid a^{b}=a^{2}\right\rangle .
$$

This is a semidirect product $G=A \rtimes B$, where $B=\langle b\rangle \cong C_{\infty}$, and $A$ is the normal closure of $a$ in $G$, an abelian group of countably infinite rank, generated by the conjugates

$$
a_{i}:=a^{2^{i}}=a^{b^{i}} \quad(i \in \mathbb{Z})
$$

of $a$, with $a_{i}^{2}=a_{i+1}$ for all $i \in \mathbb{Z}$. This subgroup $A$ can be identified with the additive group of the ring $\mathbb{Z}\left[\frac{1}{2}\right]$, with each finite product $\prod_{i} a_{i}^{e_{i}}$ in $A$ corresponding to $\sum_{i} e_{i} 2^{i} \in$ $\mathbb{Z}\left[\frac{1}{2}\right]$. Thus $a=a_{0}$ corresponds to the element $1 \in \mathbb{Z}\left[\frac{1}{2}\right]$, and $b$, acting by conjugation on $A$ as the automorphism $a_{i} \mapsto a_{i+1}$, acts on $\mathbb{Z}\left[\frac{1}{2}\right]$ by $t \mapsto 2 t$. In particular, the subgroup $H:=\langle a\rangle$ has conjugate subgroups $H_{i}:=H^{b^{-i}}=\left\langle a^{2^{-i}}\right\rangle$ for all $i \in \mathbb{Z}$, with a chain of index 2 inclusions

$$
\cdots<H_{-2}<H_{-1}<H_{0}(=H)<H_{1}<H_{2}<\cdots .
$$

Now let $\mathbb{T}$ be the Sylow 2 -subgroup of $\mathbb{Q} / \mathbb{Z}$, that is,

$$
\mathbb{T}:=\mathbb{Z}\left[\frac{1}{2}\right] / \mathbb{Z} \cong C_{2^{\infty}}:=\bigcup_{e \geq 0} C_{2^{e}}
$$

where $C_{2^{e}}$ corresponds to the subgroup generated by the image of $2^{-e}$ in $\mathbb{Z}\left[\frac{1}{2}\right] / \mathbb{Z}$. Let

$$
\Omega=\mathbb{T} \times \mathbb{Z}=\bigcup_{i \in \mathbb{Z}} \Omega_{i},
$$

the disjoint union of countably many copies $\Omega_{i}=\mathbb{T} \times\{i\}$ of $\mathbb{T}$. Let the generators $a$ and $b$ of $G$ act on $\Omega$ by

$$
a:(t, i) \mapsto\left(t+2^{i}, i\right) \quad \text { and } \quad b:(t, i) \mapsto(t, i-1)
$$

for $t \in \mathbb{T}$ and $i \in \mathbb{Z}$, where we interpret $t+2^{i}$ as an element of $\mathbb{T}$ in the obvious way. Thus $a$ preserves each set $\Omega_{i}$, fixing it pointwise for $i \geq 0$, and with cycles of length $2^{-i}$ on it for $i<0$, while $b$ induces the obvious bijection $\Omega_{i} \rightarrow \Omega_{i-1}$. Then the element $a^{b}=b^{-1} a b$ acts on $\Omega$ by a composition of three permutations

$$
a^{b}:(t, i) \mapsto(t, i+1) \mapsto\left(t+2^{i+1}, i+1\right) \mapsto\left(t+2^{i+1}, i\right)
$$

This has the same effect as

$$
a^{2}:(t, i) \mapsto\left(t+2^{i+1}, i\right),
$$

so we have a group action of $G$ on $\Omega$.

It is easy to see from the decomposition $G=A \rtimes B$ that $G$ acts faithfully and transitively on $\Omega$, that the stabiliser of the element $\alpha=(0,0) \in \Omega$ is $H=\langle a\rangle$, and that $N_{G}(H)=A$, so that

$$
N_{G}(H) / H=A / H \cong \mathbb{T} .
$$

We now calculate

$$
C:=C_{S}(G)=C_{S}(A) \cap C_{S}(B),
$$


where $S:=\operatorname{Sym}(\Omega)$. First note that $C_{S}(A)$ must permute the orbits of $A$, which are the sets $\Omega_{i}$, and must do so trivially since $A$ has a different representation, with kernel $H_{i}=$ $\left\langle a^{2^{-i}}\right\rangle$, on each $\Omega_{i}$. Since $A$ induces the regular representation of the abelian group $A / H_{i}$ on $\Omega_{i}$, it follows that $C_{S}(A)$ must be the cartesian product $\prod_{i \in \mathbb{Z}} A / H_{i}$ of the groups $A / H_{i}$, each factor $A / H_{i}$ acting regularly on $\Omega_{i}$ and fixing $\Omega \backslash \Omega_{i}$. Even though the subgroups $H_{i}$ are all distinct, we have $A / H_{i} \cong \mathbb{T}$ for all $i \in \mathbb{Z}$, so $C_{S}(A) \cong \mathbb{T}^{\mathbb{Z}}$. The only elements of $C_{S}(A)$ commuting with $b$ are those corresponding to elements of the diagonal subgroup of $\mathbb{T}^{\mathbb{Z}}$, inducing the same permutation on each subset $\Omega_{i}$. These form a group isomorphic to $\mathbb{T}$, proving that

$$
C \cong N_{G}(H) / H .
$$

Thus $G$ satisfies Theorem 1.2(1), but what about statements (2) and $\left(2^{\prime}\right)$ ? In this example, the orbits of $C$ are the sets $\Omega_{i}$, each permuted regularly by $C$, while the subset of $\Omega$ fixed by $H$ is the disjoint union

$$
\bigcup_{i \geq 0} \Omega_{i}
$$

of infinitely many of these orbits. Thus $G$ does not satisfy statement $\left(2^{\prime}\right)$. However, it does satisfy (2) since the points with stabiliser $H$ are those in $\Omega_{0}$, forming a regular orbit of $C$; the points in $\Omega_{i}$ for $i>0$, although they are also fixed by $H$, have stabiliser $H_{i}$ properly containing $H$.

Remark 4.2. This example also gives a construction of the oriented hypermap $\mathcal{H}$ with monodromy group $G$, corresponding to the epimorphism $\theta: F_{2} \rightarrow G, X \mapsto a, Y \mapsto b$. We can identify the edges with the elements $(t, i)$ of $\Omega=\mathbb{T} \times \mathbb{Z}=\cup_{i} \Omega_{i}$. The white vertices, corresponding to the cycles of $b$, can be identified with the elements $t$ of $\mathbb{T}$, each incident with the edges $(t, i)$ for $i \in \mathbb{Z}$ in decreasing order of $i$ as one follows the local orientation. The black vertices correspond to the cycles of $a$; those in $\Omega_{i}$ for $i \geq 0$ are fixed points $(t, i)$ of $a$, so they have valency 1 , each of them connected by the edge $(t, i)$ to the white vertex $t$; those in $\Omega_{i}$ for $i<0$, also denoted by $(t, i)$, correspond to cycles $\left\{\left(t+k 2^{i}, i\right) \mid k=0,1, \ldots, 2^{-i}-1\right\}$ of length $2^{-i}$, so they have valency $2^{-i}$ and are connected by edges $\left(t+k 2^{i}, i\right)$ to the white vertices $t+k 2^{i}$ in cyclic order of increasing $k$. The two faces incident with an edge $(t, i)$ have vertices, following the orientation and in alternate colours, given by

$$
\ldots,\left(t+2^{i}, i-1\right), t+2^{i},(t, i), t,(t, i+1), t-2^{i+1}, \ldots
$$

and

$$
\ldots, t+2^{i-1},(t, i-1), t,(t, i), t-2^{i},\left(t-2^{i}, i+1\right), \ldots .
$$

The group $\operatorname{Aut}_{\mathfrak{H}^{+}}(\mathcal{H})$ can be identified with $\mathbb{T}$ : each $t_{0} \in \mathbb{T}$ acts on edges of $\mathcal{H}$ by $(t, i) \mapsto\left(t+t_{0}, i\right)$, with the obvious induced actions on black and white vertices and faces. Thus it acts transitively on white vertices and on faces, whereas its orbits on black vertices and on edges consist of those in each subset $\Omega_{i}$ for $i \in \mathbb{Z}$. In particular, it acts regularly on edges (and on black vertices) in $\Omega_{0}$, those with stabiliser $H$ in $G$, but it is intransitive on those with $i \geq 0$, fixed by $H$.

It is not easy to visualise this hypermap $\mathcal{H}$. An alternative approach is to regard it as a regular branched covering, with covering group $\mathbb{T}$, of the quotient hypermap $\overline{\mathcal{H}}=$ $\mathcal{H} / \operatorname{Aut}_{\mathfrak{H}^{+}}(\mathcal{H})$ corresponding to the epimorphism $F_{2} \rightarrow G^{\text {ab }}=G / A \cong \mathbb{Z}$. As a bipartite map this is planar, with one face and one white vertex incident with 1-valent black vertices 


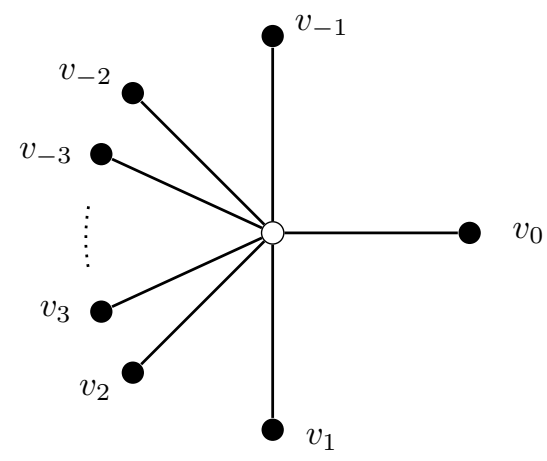

Figure 2: The hypermap $\overline{\mathcal{H}}$

$v_{i}$ in decreasing order of $i \in \mathbb{Z}$ (see Figure 2). The covering $\mathcal{H} \rightarrow \overline{\mathcal{H}}$ is branched only at the vertices $v_{i}$ for $i<0$, where the local monodromy permutation has infinitely many cycles of length $2^{-i}$, corresponding to the cycles of $b$ on $\Omega_{i}$. The automorphism group of $\mathcal{H}$ can be identified with the group of covering transformations, acting regularly on the sheets of the covering.

Remark 4.3. There is an obvious generalisation of this example based on the BaumslagSolitar group $G=B S(1, q)=\left\langle a, b \mid a^{b}=a^{q}\right\rangle$ for an arbitrary integer $q \neq 0, \pm 1$. See [8] for a discussion of the oriented hypermaps associated with the Baumslag-Solitar groups $B S(p, q)=\left\langle a, b \mid\left(a^{p}\right)^{b}=a^{q}\right\rangle$ for arbitrary $p, q \neq 0$.

\section{Primitive monodromy groups}

If $G$ is a permutation group on a set $\Omega$, then the relation $G_{\alpha}=G_{\beta}$, appearing in Theorem 1.2 via the definition of $\Phi$, is a $G$-invariant equivalence relation on $\Omega$, and its equivalence classes are the orbits of the centraliser $C$ of $G$. Recall that a permutation group is primitive if it preserves no non-trivial equivalence relation; equivalently, the point-stabilisers are maximal subgroups. As an immediate consequence of Theorem 1.2, we hav

Corollary 5.1. If $G$ is a primitive permutation group, then either $G \cong C_{p}$, acting regularly, for some prime $p$, with centraliser $C=G$, or the centraliser $C$ of $G$ is the trivial group.

Proof. The equivalence relation $G_{\alpha}=G_{\beta}$ on $\Omega$ must be either the identity or the universal relation. In the first case the equivalence classes are singletons, so $|C|=1$. In the second case $G_{\alpha}=\{1\}$; this is a maximal subgroup of $G$, so $G \cong C_{p}$ for some prime $p$, with $C=G$.

Corollary 5.2. In a permutational category $\mathfrak{C}$, if the monodromy group $G$ of an object $\mathcal{O}$ is a primitive permutation group, then either $\mathcal{O}$ is regular, with $\operatorname{Aut}_{\mathfrak{C}}(\mathcal{O})=G \cong C_{p}$ for some prime $p$, or $\operatorname{Aut}_{\mathfrak{C}}(\mathcal{O})$ is the trivial group.

Of course, there are many examples of primitive permutation groups, either sporadic or members of infinite families: just represent a group on the cosets of a maximal subgroup. On the other hand, Cameron, Neumann and Teague [3] have shown that for a set of integers 
$n \in \mathbb{N}$ of asymptotic density 1 the only primitive groups of degree $n$ are the alternating and symmetric groups $A_{n}$ and $S_{n}$.

Example 5.3. The symmetric and alternating groups, in their natural representationas, arise quite frequently as monodromy groups in various categories. For instance, let $\mathfrak{C}=\mathfrak{H}^{+}$, the category of oriented hypermaps, with parent group $\Gamma=F_{2}=\langle X, Y \mid-\rangle$. A theorem of Dixon [4] states that a randomly chosen pair of permutations $x, y \in S_{n}$ generate $S_{n}$ or $A_{n}$ with probability approaching $3 / 4$ or $1 / 4$ as $n \rightarrow \infty$, so in that sense 'most' finite objects in this category have a symmetric or alternating monodromy group, and a trivial automorphism group.

In most of the permutational categories of current interest, it is simple to describe the regular objects with automorphism group $C_{p}$ for each prime $p$; apart from these exceptions, objects with a primitive monodromy group have a trivial automorphism group. The exceptions correspond to the normal subgroups of index $p$ in the parent group $\Gamma$, or equivalently to the maximal subgroups in the elementary abelian $p$-group $\Gamma / \Gamma^{\prime} \Gamma^{p}$, where $\Gamma^{\prime}$ and $\Gamma^{P}$ are the subgroups of $\Gamma$ generated by the commutators and $p$-th powers. In the categories listed in $\S 2$, they are as follows.

If $\mathfrak{C}=\mathfrak{H}^{+}$or $\mathfrak{D}$ then $\Gamma=F_{2}$, so $\Gamma / \Gamma^{\prime} \Gamma^{p} \cong C_{p} \times C_{p}$, with $p+1$ maximal subgroups. Of the corresponding oriented hypermaps, three have type a permutation of $(p, p, 1)$ and are planar, while the remaining $p-2$ have type $(p, p, p)$ and genus $(p-1) / 2$. As dessins, the former are on the Riemann sphere, with Belyi functions $\beta: z \mapsto z^{p}, 1 /\left(1-z^{p}\right)$ and $1-z^{-p}$, and automorphisms $z \mapsto \zeta z$ where $\zeta^{p}=1$. The latter are on Lefschetz curves $y^{p}=x^{u}(x-1)$ for $u=1, \ldots, p-2$, each with a Belyi function $\beta:(x, y) \mapsto x$ and automorphisms $(x, y) \mapsto(x, \zeta y)$ where $\zeta^{p}=1$ (see [12, Example 5.6]). The four dessins for $p=3$ are shown in Figure 3; in the dessin on the right, opposite sides of the hexagon are identified to form a torus.
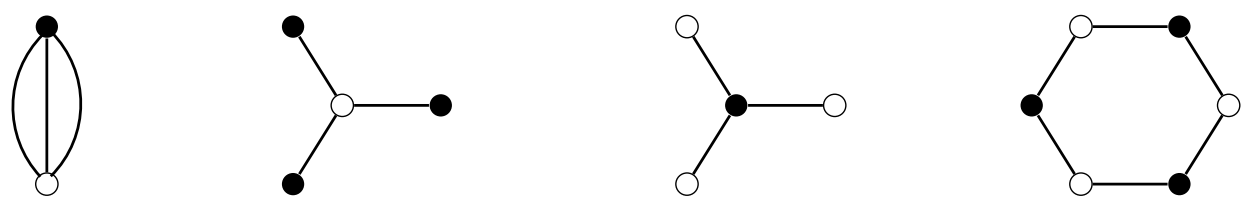

Figure 3: The four dessins with primitive monodromy group $C_{p}, p=3$

If $\mathfrak{C}=\mathfrak{M}^{+}$then $\Gamma=C_{\infty} * C_{2}$, so $\Gamma / \Gamma^{\prime} \Gamma^{p} \cong V_{4}$ or $C_{p}$ as $p=2$ or $p>2$, giving three oriented maps or one, all planar. Their types are the three permutations of $(2,2,1)$, together with $(p, 1, p)$. They are shown, for $p=2$ and 3 , in Figure 4.
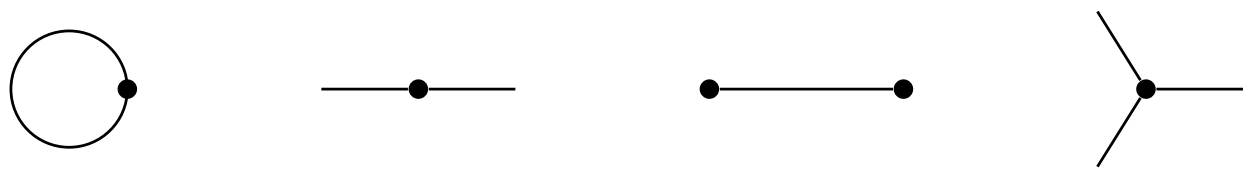

Figure 4: The four oriented maps with primitive monodromy group $C_{p}, p=2,3$ 


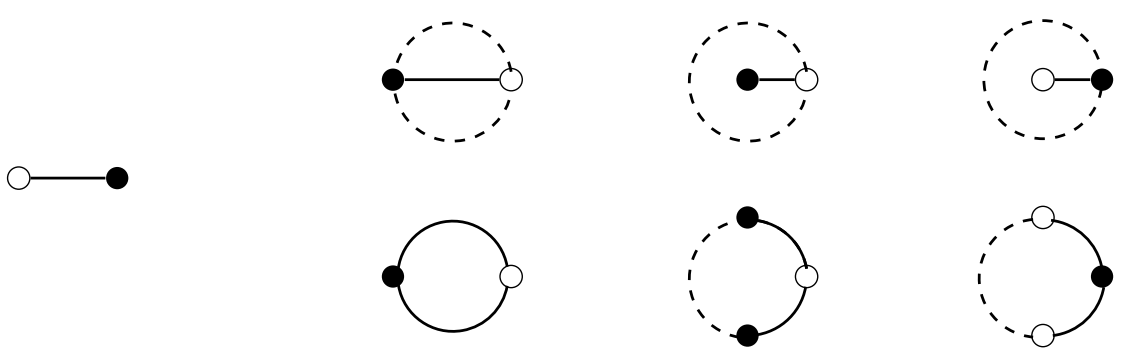

Figure 5: The seven hypermaps with primitive monodromy group $C_{2}$

If $\mathfrak{C}=\mathfrak{H}$ or $\mathfrak{M}$ then $\Gamma=C_{2} * C_{2} * C_{2}$ or $V_{4} * C_{2}$, so in either case $\Gamma / \Gamma^{\prime} \Gamma^{p} \cong V_{8}$ or 1 as $p=2$ or $p>2$. If $p=2$ there are seven hypermaps and seven maps; if $p>2$ there are none. The hypermaps are shown in Figure 5. The hypermap on the left is planar, while the other six are on the closed disc, shown by a broken line. The seven maps can be obtained from these hypermaps by ignoring all the white vertices.

If $X$ is a compact orientable surface of genus $g$ then there are $\left(p^{2 g}-1\right) /(p-1)$ regular coverings $Y \rightarrow X$ with monodromy group $C_{p}$, corresponding to the normal subgroups of index $p$ in $\pi_{1} X=\left\langle A_{i}, B_{i}(i=1, \ldots, g) \mid \prod_{i}\left[A_{i}, B_{i}\right]=1\right\rangle$; the surfaces $Y$ are orientable, of genus $1+p(g-1)$. In the non-orientable case, with $\pi_{1} X=\left\langle R_{i}(i=1, \ldots, g)\right.$ $\left.\prod_{i} R_{i}^{2}=1\right\rangle$ there are $\left(p^{g-1}-1\right) /(p-1)$ or $2^{g}-1$ such coverings as $p>2$ or $p=2$; the surfaces $Y$ are all non-orientable, of genus $2+p(g-2)$, apart from the orientable double cover of $X$ for $p=2$, which has genus $g-1$.

\section{Automorphism groups of non-connected objects}

It is often convenient to restrict attention to the connected objects in a category, as we have done so far. Here we will briefly show how Theorem 1.1 extends to non-connected objects.

If $\mathcal{C}$ is a permutational category with parent group $\Gamma$, then the connected components $\mathcal{O}_{i}(i \in I)$ of an object $\mathcal{O}$ in $\mathfrak{C}$ correspond bijectively to the orbits $\Omega_{i}(i \in I)$ of $\Gamma$ on the set $\Omega$ associated with $\mathcal{O}$. As before, $\operatorname{Aut}_{\mathfrak{C}}(\mathcal{O})$ is isomorphic to the centraliser $C$ of $\Gamma$ in $S=\operatorname{Sym}(\Omega)$. In order to describe the structure of $C$ in general, we first consider two extreme cases.

Suppose first that the components $\mathcal{O}_{i}$ are mutually non-isomorphic. This is equivalent to the point stabilisers $M_{i} \leq \Gamma$ for different orbits $\Omega_{i}$ being mutually non-conjugate in $\Gamma$. Then $C$ is the cartesian product of the centralisers $C_{i} \leq \operatorname{Sym}\left(\Omega_{i}\right)$ of $\Gamma$ on the sets $\Omega_{i}$. By the transitivity of $\Gamma$ on $\Omega_{i}$, we have $C_{i} \cong N_{G_{i}}\left(H_{i}\right) \cong N_{\Gamma}\left(M_{i}\right) / M_{i}$ for each $i \in I$, where $G_{i}$ is the permutation group induced by $\Gamma$ on $\Omega_{i}$, and $H_{i}$ is a point stabiliser in $G_{i}$ for this action.

At the other extreme, suppose that the components $\mathcal{O}_{i}$ are all isomorphic, or equivalently the point stabilisers $M_{i}$ are conjugate to each other. Then $C$ is the wreath product $C_{i}$ (Sym $(I)$ of $C_{i}$ by $\operatorname{Sym}(I)$. This is a semidirect product, in which the normal subgroup is the cartesian product of the mutually isomorphic groups $C_{i}\left(\cong N_{G_{i}}\left(H_{i}\right) \cong N_{\Gamma}\left(M_{i}\right) / M_{i}\right)$ for $i \in I$, and the complement is $\operatorname{Sym}(I)$, acting on this normal subgroup by permuting the factors $C_{i}$ via isomorphisms between them.

We can now describe the general form of $C$ by combining these two constructions. 
We first partition the set of components of $\mathcal{O}$ into maximal sets $\left\{\mathcal{O}_{i j} \mid i \in I_{j}\right\}(j \in J)$ of mutually isomorphic objects $\mathcal{O}_{i j}$, each subset indexed by a set $I_{j}$. We then define $C_{i j} \cong$ $N_{G_{i j}}\left(H_{i j}\right) \cong N_{\Gamma}\left(M_{i j}\right) / M_{i j}$ with obvious notation) to be the centraliser of $\Gamma$ in $\operatorname{Sym}\left(\Omega_{i j}\right)$. Then $C$, and hence also $\operatorname{Aut}_{\mathfrak{C}}(\mathcal{O})$, is the cartesian product over all $j \in J$ of the wreath products $C_{i j} 2 \operatorname{Sym}\left(I_{j}\right)$ where $i \in I_{j}$. This is again a semidirect product, where the normal subgroup is the cartesian product of all the groups $C_{i j}\left(i \in I_{j}, j \in J\right)$, and the complement is the cartesian product of the groups $\operatorname{Sym}\left(I_{j}\right)(j \in J)$, each factor $\operatorname{Sym}\left(I_{j}\right)$ of the latter acting on the normal subgroup by permuting the factors $C_{i j}$ for $i \in I_{j}$ while fixing all other factors.

This description can be used to determine the cardinality of $\operatorname{Aut}_{\mathfrak{C}}(\mathcal{O})$. We will restrict attention to categories where the parent group $\Gamma$ is countable (for instance, where it is finitely generated), since this condition is satisfied by most of the examples studied; the modifications required for an uncountable parent group are straightforward. By Theorem 1.1 this implies that $\operatorname{Aut}_{\mathfrak{C}}(\mathcal{O})$ is also countable for each connected object $\mathcal{O}$. Since a cartesian product of infinitely many non-trivial groups is uncountable, as is the symmetric group on any infinite set, the following is clear:

Theorem 6.1. Let $\mathfrak{C}$ be a permutational category for which the parent group $\Gamma$ is countable, and let $\mathcal{O}$ be an object in $\mathfrak{C}$ with connected components $\mathcal{O}_{i j}$, indexed by sets $I_{j}(j \in J)$ as above. Then

1. $\left|\operatorname{Aut}_{\mathfrak{C}}(\mathcal{O})\right|>\aleph_{0}$ if and only if either $\mathcal{O}$ has infinitely many components $\mathcal{O}_{i j}$ with $\left|\operatorname{Aut}_{\mathfrak{C}}\left(\mathcal{O}_{i j}\right)\right|>1$, or at least one set $I_{j}$ is infinite;

2. $\left|\operatorname{Aut}_{\mathfrak{C}}(\mathcal{O})\right|=\aleph_{0}$ if and only if $\mathcal{O}$ has only finitely many components $\mathcal{O}_{i j}$ with $\left|\operatorname{Aut}_{\mathfrak{C}}\left(\mathcal{O}_{i j}\right)\right|>1$, each set $I_{j}$ is finite, and $\operatorname{Aut}_{\mathfrak{C}}\left(\mathcal{O}_{i j}\right)$ is infinite for some component $\mathcal{O}_{i j}$;

3. $\left|\operatorname{Aut}_{\mathfrak{C}}(\mathcal{O})\right|<\aleph_{0}$ if and only if $\mathcal{O}$ has only finitely many components $\mathcal{O}_{i j}$ with $\left|\operatorname{Aut}_{\mathfrak{C}}\left(\mathcal{O}_{i j}\right)\right|>1$, each set $I_{j}$ is finite, and $\operatorname{Aut}_{\mathfrak{C}}\left(\mathcal{O}_{i j}\right)$ is finite for each component $\mathcal{O}_{i j}$.

Corollary 6.2. In Case (3), where $\operatorname{Aut}_{\mathfrak{C}}(\mathcal{O})$ is finite, it has order

$$
\prod_{j \in J}\left|\operatorname{Aut}_{\mathfrak{C}}\left(\mathcal{O}_{i j}\right)\right|^{\left|I_{j}\right|}\left|I_{j}\right| ! .
$$

Example 6.3. Let $\mathfrak{C}=\mathfrak{M}^{+}$, the category of oriented maps, which has parent group $\Gamma=$ $\Delta(\infty, 2, \infty)=\left\langle X, Y \mid Y^{2}=1\right\rangle$. For each integer $n \geq 2$ let $\tilde{\mathcal{M}}_{n}$ be the minimal regular cover of the map $\mathcal{M}_{n} \in \mathfrak{M}^{+}$in Figure 1. This is a regular oriented map with automorphism and monodromy group $S_{n}$ (in its regular representation) corresponding to the epimorphism $\Gamma \rightarrow S_{n}, X \mapsto(1,2, \ldots, n), Y \mapsto(1,2)$. If we take $\mathcal{M}$ to be the disjoint union of these maps $\tilde{\mathcal{M}}_{n}$, then Aut $_{\mathfrak{M}^{+}}(\mathcal{M})$ is the cartesian product $\prod_{n \geq 2} S_{n}$. This uncountable group is very rich in subgroups: for instance, every finitely generated residually finite group (such as every finitely generated linear group, by Mal'cev's Theorem [14]) can be embedded in a cartesian product of finite groups of distinct orders, and hence (by Cayley's Theorem) can be embedded in $\operatorname{Aut}_{\mathfrak{M}^{+}}(\mathcal{M})$.

\section{Morphisms}

Although this paper has concentrated on automorphisms, Theorem 1.1 can be extended to describe the morphisms $\mathcal{O}_{1} \rightarrow \mathcal{O}_{2}$ between connected objects. If each $\mathcal{O}_{i}$ corresponds to 
an action of the parent group $\Gamma$ on $\Omega_{i}$, with stabiliser $M_{i}$, then such a morphism exists if and only if $M_{1}$ is conjugate to a subgroup of $M_{2}$. The set $B:=\left\{b \in \Gamma \mid M_{1}^{b} \leq M_{2}\right\}$ is a union of cosets $b M_{2}(b \in B)$, and these correspond bijectively to the morphisms $\mathcal{O}_{1} \rightarrow \mathcal{O}_{2}$. Specifically, if we identify elements of $\Omega_{i}$ with cosets $M_{i} g(g \in \Gamma)$, then each $b \in B$ corresponds to the morphism $\phi_{b}: M_{1} g \mapsto M_{2} b^{-1} g$, where $\phi_{b}=\phi_{b^{\prime}}$ if and only if $b^{\prime} \in b M_{2}$. The proof of this in [11, Theorem 3.5] for oriented maps extends easily to all permutational categories. This shows that morphisms between connected objects are always surjective, but Example 5 shows that endomorphisms $\mathcal{O} \rightarrow \mathcal{O}$ of infinite objects need not be automorphisms. This also shows that the number of morphisms $\mathcal{O}_{1} \rightarrow \mathcal{O}_{2}$ is bounded above by $\left|\Omega_{2}\right|=\left|\Gamma: M_{2}\right|$, attained if (and only if, when $\mathcal{O}_{2}$ is finite) $M_{1}$ is contained in the core of $M_{2}$.

Example 7.1. If $\mathcal{O}_{i}$ is regular for $i=1$ or 2 , so that $M_{i}$ is a normal subgroup of $\Gamma$, then $B=\Gamma$ or $\emptyset$ as $M_{1} \leq M_{2}$ or not, and there are respectively $\left|\Omega_{2}\right|$ morphisms $\mathcal{O}_{1} \rightarrow \mathcal{O}_{2}$ or none.

There is an action of $\operatorname{Aut}_{\mathfrak{C}}\left(\mathcal{O}_{1}\right) \times \operatorname{Aut}_{\mathfrak{C}}\left(\mathcal{O}_{2}\right)$ on the set $\operatorname{Mor}_{\mathfrak{C}}\left(\mathcal{O}_{1}, \mathcal{O}_{2}\right)$ of morphisms $\mathcal{O}_{1} \rightarrow \mathcal{O}_{2}$, given by $\left(\theta_{1}, \theta_{2}\right): \phi \mapsto \theta_{1}^{-1} \circ \phi \circ \theta_{2}$. To realise this action concretely, note that the action $\left(n_{1}, n_{2}\right): b \mapsto n_{1}^{-1} b n_{2}$ of $N_{\Gamma}\left(M_{1}\right) \times N_{\Gamma}\left(M_{2}\right)$ on $B$ yields an induced action on the set of orbits $b M_{2} \subseteq B$ of its normal subgroup $1 \times M_{2}$, with $M_{1} \times M_{2}$ in the kernel; the resulting action of $\left(N_{\Gamma}\left(M_{1}\right) \times N_{\Gamma}\left(M_{2}\right)\right) /\left(M_{1} \times M_{2}\right) \cong N_{\Gamma}\left(M_{1}\right) / M_{1} \times N_{\Gamma}\left(M_{2}\right) / M_{2}$ on these orbits is equivalent to the action of $\operatorname{Aut}_{\mathfrak{C}}\left(\mathcal{O}_{1}\right) \times \operatorname{Aut}_{\mathfrak{C}}\left(\mathcal{O}_{2}\right)$ on $\operatorname{Mor}_{\mathfrak{C}}\left(\mathcal{O}_{1}, \mathcal{O}_{2}\right)$. Thus if $\theta_{i}$ is given by $M_{i} g \mapsto n_{i}^{-1} M_{i} g$ for $i=1,2$, then $\left(\theta_{1}, \theta_{2}\right): \phi_{b} \mapsto \theta_{1}^{-1} \circ \phi_{b} \circ \theta_{2}=\phi_{b^{\prime}}$, where $b^{\prime}=n_{1}^{-1} b n_{2} \in B$.

The subgroup of $\operatorname{Aut}_{\mathfrak{C}}\left(\mathcal{O}_{1}\right) \times \operatorname{Aut}_{\mathfrak{C}}\left(\mathcal{O}_{2}\right)$ fixing a morphism $\phi$ consists of those pairs $\left(\theta_{1}, \theta_{2}\right)$ such that $\theta_{1} \circ \phi=\phi \circ \theta_{2}$, that is, $\phi$ lifts $\theta_{2}$ to $\theta_{1}$. In particular, the subgroup of Aut $_{\mathfrak{C}}\left(\mathcal{O}_{1}\right)$ fixing a morphism $\phi$ consists of the covering transformations of $\phi$. Similarly, the action of $\operatorname{Aut}_{\mathfrak{C}}\left(\mathcal{O}_{2}\right)$ on $\operatorname{Mor}_{\mathfrak{C}}\left(\mathcal{O}_{1}, \mathcal{O}_{2}\right)$ is equivalent to its action on $\Omega_{2}$ : it is always semi-regular, and regular if and only if $\mathcal{O}_{2}$ is regular.

\section{ORCID iDs}

Gareth Aneurin Jones (DD https://orcid.org/0000-0002-7082-7025

\section{References}

[1] G. Baumslag and D. Solitar, Some two-generator one-relator non-Hopfian groups, Bull. Amer. Math. Soc. 68 (1962), 199-201, doi:10.1090/s0002-9904-1962-10745-9.

[2] G. V. Bely̆, On Galois extensions of a maximal cyclotomic field, Mathematics of the USSRIzvestiya 14 (1980), 247-256, doi:10.1070/im1980v014n02abeh001096.

[3] P. J. Cameron, P. M. Neumann and D. N. Teague, On the degrees of primitive permutation groups, Math. Z. 180 (1982), 141-150, doi:10.1007/bf01318900.

[4] J. D. Dixon, The probability of generating the symmetric group, Math. Z. 110 (1969), 199-205, doi:10.1007/bf01110210.

[5] R. Frucht, Herstellung von Graphen mit vorgegebener abstrakter Gruppe, Compositio Math. 6 (1939), 239-250, http://www. numdam.org/item?id=CM_1939_6_239_0.

[6] E. Girondo and G. González-Diez, Introduction to compact Riemann surfaces and dessins d'enfants, volume 79 of London Mathematical Society Student Texts, Cambridge University Press, Cambridge, 2012, doi:10.1017/cbo9781139048910. 
[7] A. Grothendieck, Esquisse d'un programme (1984), in: Geometric Galois actions, 1, Cambridge Univ. Press, Cambridge, volume 242 of London Math. Soc. Lecture Note Ser, pp. 5-47, 1997, with an English translation on pp. 243-283, https://webusers.imj-prg.fr/ reila.schneps/grothendieckcircle/.

[8] G. A. Jones, Exotic behaviour of infinite hypermaps, Ars Math. Contemp. 1 (2008), 51-65, doi:10.26493/1855-3974.24.035.

[9] G. A. Jones, Combinatorial categories and permutation groups, Ars Math. Contemp. 10 (2016), 237-254, doi:10.26493/1855-3974.545.fd5.

[10] G. A. Jones, Realisation of groups as automorphism groups in categories, 2018, https:// arxiv.org/abs/1807.00547.

[11] G. A. Jones and D. Singerman, Theory of maps on orientable surfaces, Proc. London Math. Soc. (3) 37 (1978), 273-307, doi:10.1112/plms/s3-37.2.273.

[12] G. A. Jones and J. Wolfart, Dessins d'enfants on Riemann surfaces, Springer Monographs in Mathematics, Springer, Cham, 2016, doi:10.1007/978-3-319-24711-3.

[13] S. K. Lando and A. K. Zvonkin, Graphs on surfaces and their applications, volume 141 of Encyclopaedia of Mathematical Sciences, Springer-Verlag, Berlin, 2004, doi:10.1007/ 978-3-540-38361-1, with an appendix by Don B. Zagier, Low-Dimensional Topology, II.

[14] A. Malcev, On isomorphic matrix representations of infinite groups, Rec. Math. [Mat. Sbornik] N.S. 8 (50) (1940), 405-422.

[15] W. S. Massey, Algebraic topology: An introduction, Harcourt, Brace \& World, Inc., New York, 1967.

[16] P. McMullen and E. Schulte, Abstract regular polytopes, volume 92 of Encyclopedia of Mathematics and its Applications, Cambridge University Press, Cambridge, 2002, doi:10.1017/ cbo9780511546686.

[17] J. R. Munkres, Topology, Prentice Hall, Inc., Upper Saddle River, NJ, 2000, second edition of [MR0464128].

[18] H. Neumann, Varieties of Groups, Springer-Verlag, Berlin - Heidelberg, 1st edition, 1967, doi: 10.1007/978-3-642-88599-0.

[19] C. E. Praeger and C. Schneider, Permutation groups and Cartesian decompositions, volume 449 of London Mathematical Society Lecture Note Series, Cambridge University Press, Cambridge, 2018, doi:10.1017/9781139194006.

[20] G. Sabidussi, Graphs with given infinite group, Monatsh. Math. 64 (1960), 64-67, doi:10.1007/ bf01319053.

[21] T. R. S. Walsh, Hypermaps versus bipartite maps, J. Combinatorial Theory Ser. B 18 (1975), 155-163, doi:10.1016/0095-8956(75)90042-8. 